Test Method

\title{
Mapping internal deformation fields in 3D printed porous structure with digital volume correlation
}

\author{
Bo Wang ${ }^{\mathrm{a}}$, Lijuan Sun ${ }^{\mathrm{b}}$, Bing Pan ${ }^{\mathrm{a}, *}$ \\ ${ }^{a}$ Institute of Solid Mechanics, Beihang University, Beijing, 100191, China \\ ${ }^{\mathrm{b}}$ Institute of Mechanics, Chinese Academy of Sciences, Beijing, 100190, China
}

\section{A R T I C L E I N F O}

\section{Keywords:}

3D printed porous structure

Digital volume correlation

X-ray tomographic imaging

\begin{abstract}
A B S T R A C T
As a new manufacturing technology, 3D printing is gradually attracting more interest in both the academic community and the industrial field. To realize its full potential in engineering applications, it is necessary to characterize the mechanical behaviors and properties of 3D printed materials and structures under external loading. Although surface deformation of 3D printed materials has been extensively investigated, the 3D internal deformation measurement and further mechanical analysis using digital volume correlation (DVC) gain less attention in the field of 3D printing. In this work, step-wise uniaxial compression tests of a 3D printed porous structure were first performed in combination with in-situ X-ray computed tomography (CT) imaging. Then, the global and local evolutions of internal full-field deformation at different compressive stages were evaluated via DVC technique. Finally, a multiscale experimental framework was presented to correlate mechanical properties with geometric structure of the 3D printed porous structure. On this basis, the failure patterns along with the possible damage mechanism were predicted and discussed.
\end{abstract}

\section{Introduction}

Engineering application of modern materials and structures relies on advanced manufacturing techniques and novel experimental protocols to realize specific structural and functional properties. The emergence of 3D printing technology [1] enables the design and fabrication of materials and structures with desirable mechanical properties, which are fulfilled by fabricating specially-designed micro-structures or morphologies rather than changing material constituents. Furthermore, to characterize their mechanical performance, a thorough understanding of their internal microstructural features and the morphologymechanics relationship with the aid of advanced volumetric imaging technology is essential. To this purpose, X-ray micro-CT imaging technology has been widely employed for volumetric dimensional measurement and porosity analysis of 3D printed materials $[2,3]$.

Most 3D printed materials possess complex internal microstructures at the microscale, thus often exhibiting heterogeneous properties at the macroscale. In this case, existing techniques for surface deformation measurement only provides limited or even unreliable information on the structure-function relationship of 3D printed materials [4-14]. To shed more light on their mechanical performance, experimental techniques based on X-ray micro-CT imaging are favorable to better disclose the influence of the local morphological evolution on their macroscopic response under external loading. However, full-field internal deformation measurement and mechanical analysis of 3D printed structures still attracted little attention to date $[15,16]$.

Digital volume correlation (DVC), which was extended from 2D digital image correlation (DIC) for surface strain mapping [17,18], has experienced rapid growth in recent years as a powerful experimental technique for full-field internal deformation measurement [19-21]. In combination with advanced X-ray CT imaging technique, DVC eliminates the limitations of classical optical methods for surface deformation measurement by retrieving the interior deformation field of a material or structure under external loading. Moreover, the measured 3D internal deformation fields are able to give more insight into the relationship between internal microstructure and macroscopic mechanical properties of diverse materials or structures. More importantly, DVC measurements not only allow validation of FEM simulations and modeling but also help to provide guidance for the microstructural design of modern materials with significantly improved mechanical properties.

In this work, step-wise uniaxial compression tests of a 3D printed porous polymer sample were performed and investigated with the powerful DVC method. First, corresponding volume images at each loading stage were scanned by a lab X-ray micro-CT system. Afterward, an advanced subvolume-based DVC approach was implemented to

\footnotetext{
* Corresponding author.

E-mail address: panb@buaa.edu.cn (B. Pan).
} 


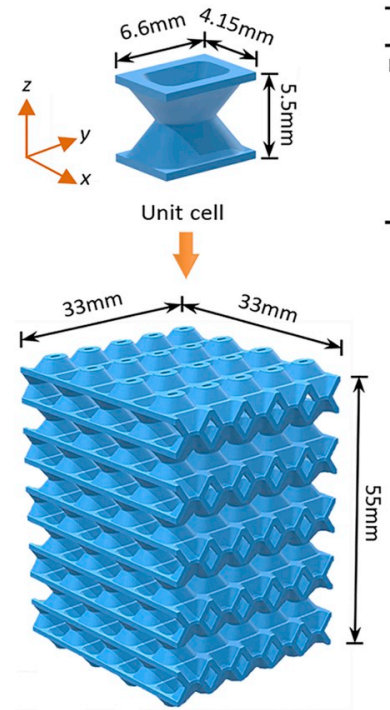

(a) Model of porous sample

\begin{tabular}{|c|c|c|c|}
\hline \multicolumn{2}{|c|}{ Properties of the printing material } & \multicolumn{2}{|c|}{ Settings of the stereolithography process } \\
\hline Heat distortion temperature & $145^{\circ} \mathrm{C}$ & Print precision & $0.1 \mathrm{~mm}$ \\
\hline Fusion point & $183^{\circ} \mathrm{C}$ & Layer thickness & $0.05 \mathrm{~mm}$ \\
\hline Tensile strength & $46 \mathrm{MPa}$ & Z-axis positioning accuracy & $0.008 \mathrm{~mm}$ \\
\hline Tensile modulus & $1602 \mathrm{MPa}$ & Scanning speed & $6 \mathrm{~m} / \mathrm{s}$ \\
\hline Fracture elongation & $36 \%$ & Spot diameter & $0.12 \mathrm{~mm}$ \\
\hline
\end{tabular}

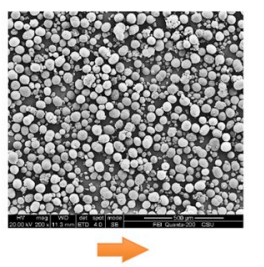

3D printing material

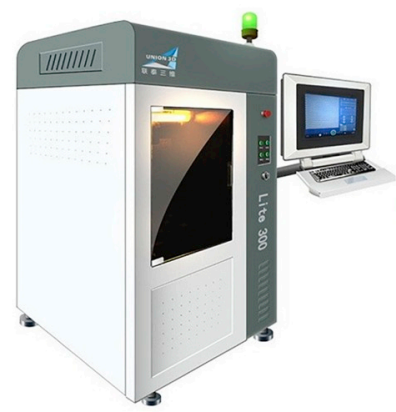

(b) 3D printer (UnionTech)

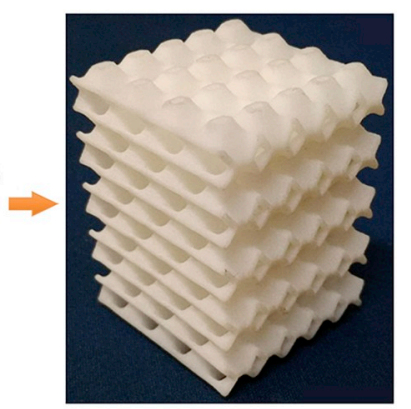

(c) 3D printed porous sample

Fig. 1. 3D geometric model and 3D printed porous sample with the inserted tables showing properties of the printing material and settings of the stereolithography process.

quantify the internal displacement and strain fields of the test sample at different loading stages. Based on in-situ X-ray CT imaging and DVC technique, an experimental framework was presented to explore 3D structural and mechanical properties of 3D printed structures. Based on this framework, both the global and local deformation responses of the test sample under step-wise compression were quantified. Also, the mechanical properties of the test sample were estimated and closely related to its structural features. Finally, the failure positions were localized and the possible damage mechanism was discussed.

\section{Experimental procedure}

\subsection{Sample preparation}

As shown in Fig. 1, the 3D geometric model of a periodically stacked hourglass structure (single cell size: $4.15 \mathrm{~mm} \times 6.6 \mathrm{~mm} \times 5.5 \mathrm{~mm}$ ) was first designed by Catia software (version: V5R20, Dassault Systèmes, France), then three porous samples with a size of $33 \mathrm{~mm} \times 33 \mathrm{~mm} \times 55 \mathrm{~mm}$ were printed using a 3D printer (Lite 300, UnionTech, China) based on stereolithography technique. The employed printing material is nylon powder (FS3300PA, Farsoon Technologies, China). During 3D printing, the 3D geometric model was first split along the z-axis into xoy horizontal slices with a thickness of $0.05 \mathrm{~mm}$. The print direction was along the longitudinal direction of the designed structure. Each successive layer polymerizes according to the geometrical model within the corresponding slice. After 3D printing was completed, sandpapers were applied to manually remove any protrusions or remaining powder on the outer surfaces. The thermal/mechanical properties of the printing material and the detailed settings of the stereolithography process are listed in the inserted table in Fig. 1.

\subsection{In-situ loading and $X$-ray CT imaging}

First, baseline rescan tests using two successive scans of the 3D printed porous sample were performed to quantify the measurement errors (displacement and strain uncertainties) associated with the volumetric imaging system and DVC algorithm. Afterward, step-wise uniaxial compression tests of the 3D printed porous sample were carried out. The experimental setup of the step-wise uniaxial compression tests, the applied time-displacement curve and displacment-force curve are exhibited in Fig. 2. A load cell (inner diameter: $80 \mathrm{~mm}$, height: $125 \mathrm{~mm}$, displacement loading precision: $0.005 \mathrm{~mm}$, force loading precision: $0.01 \mathrm{~N}$ ) was adopted for loading. Prior to the compression tests, a preset compressive load of $10 \mathrm{~N}$ was exerted on the specimen to ensure the stability of in-situ X-ray CT imaging. The step-wise compressive strains were preset as $4 \%(2.2 \mathrm{~mm})$ to $20 \%(11.0 \mathrm{~mm})$ at an increment of $4 \%(2.2 \mathrm{~mm})$ and a loading speed of $1.0 \mathrm{~mm} / \mathrm{min}$ (i.e., a strain rate of $0.03 \%$ ).

After each loading, a dwell time of 5 min was preset to avoid image blurring caused by stress relaxation of the polymer sample. Then, an X-ray micro-CT system (YXLON CT modular, YXLON International, Germany) was employed for volumetric imaging at each compressive stage. During the micro-CT scan, the voltage and current of the X-ray source $(225 \mathrm{KV} \mathrm{mi-}$ crofocus X-ray tube) were set as $140 \mathrm{kV}$ and $0.62 \mathrm{~mA}$, respectively. The sampling grid of the X-ray detector (YXLON XRD 1620) was chosen as $1000 \times 1024$ pixels with an exposure time of $350 \mathrm{~ms}$. 1080 projection images of the sample were recorded at a rotation step of $0.33^{\circ}$ and an averaging frame of 4 , resulting in a total scan time of $25.2 \mathrm{~min}$ per stage. Finally, one reference and five deformed volume images with a size of $1000 \times 1000 \times 1024$ voxels were reconstructed by the Volume Graphics Studio software (version: 3.0 ) at a voxel size of $55.3 \mu \mathrm{m}$.

\subsection{Digital volume correlation}

To extract interior full-field internal deformation in the five compressive stages, the five deformed volume images were successively compared with the reference one using an advanced subvolume-based DVC method. Before DVC analysis, a Gaussian prefilter (kernel size: $3 \times 3 \times 3$ voxels) was applied to the volume image sequence to suppress the unfavorable effect of image noise [22]. Then, a Huang's fuzzy thresholding algorithm was adopted for volume image segmentation and subsequent DVC analysis [23]. During displacement tracking, a cubic volume of interest (VOI) was first defined with a size of $600 \times 600 \times 585$ voxels (local domain: $\sim 33.2 \mathrm{~mm} \times 33.2 \mathrm{~mm} \times 32.4 \mathrm{~mm})$ and a gird step of 5 voxels $(\sim 0.28 \mathrm{~mm})$, resulting in 461490 discrete calculation points. Then, subvolume sizes ranging from 21 to 61 voxels at an increment of 10 voxels were specified at each calculation point. Finally, a 3D pointwise least-square (PLS) approach was employed to extract the six Cauchy strain fields according to the measured displacement fields in each loading stage. The strain window size was defined as $7 \times 7 \times 7$ points (local domain: $\sim 1.936 \mathrm{~mm} \times 1.936 \mathrm{~mm} \times 1.936 \mathrm{~mm}$ ) during strain mapping. A cropped reference volume image, the defined volume of interest and their intensity histograms are shown Fig. 3. 


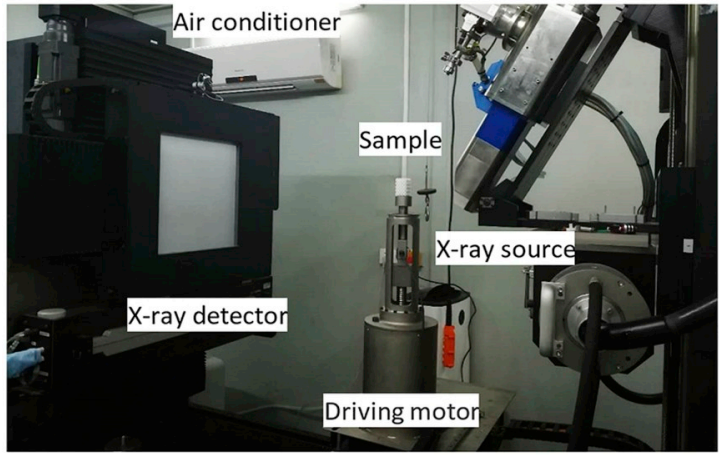

(a) Micro X-ray CT device

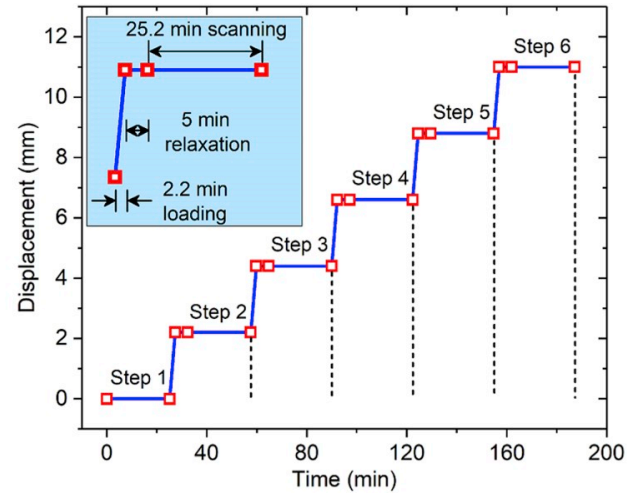

(b) Time-displacement curve

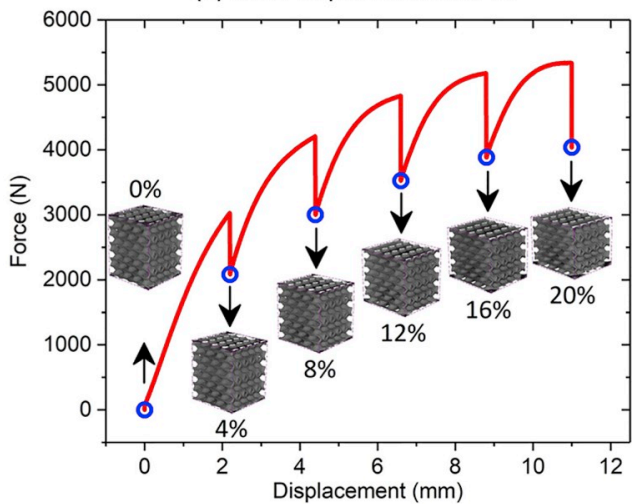

(c) Displacement-force curve

Fig. 2. (a) Experimental setup, (b) time-displacement curve and (c) displacement-force curve in the step-wise compression tests.

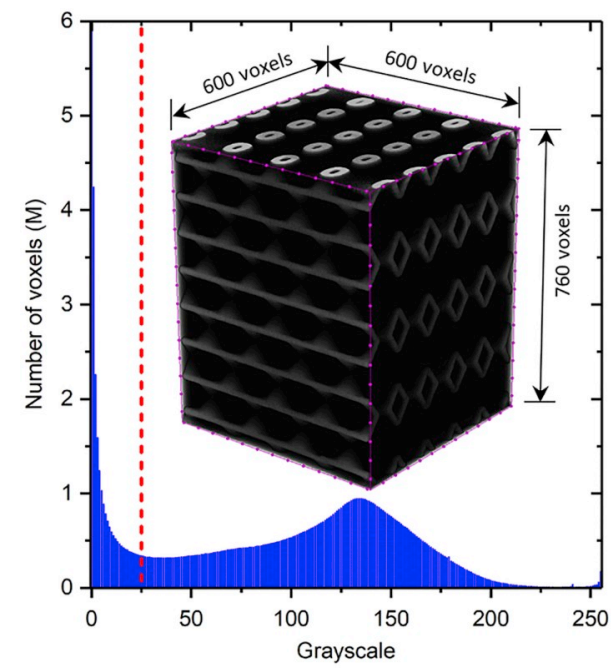

(a) Cropped reference volume image and its histogram

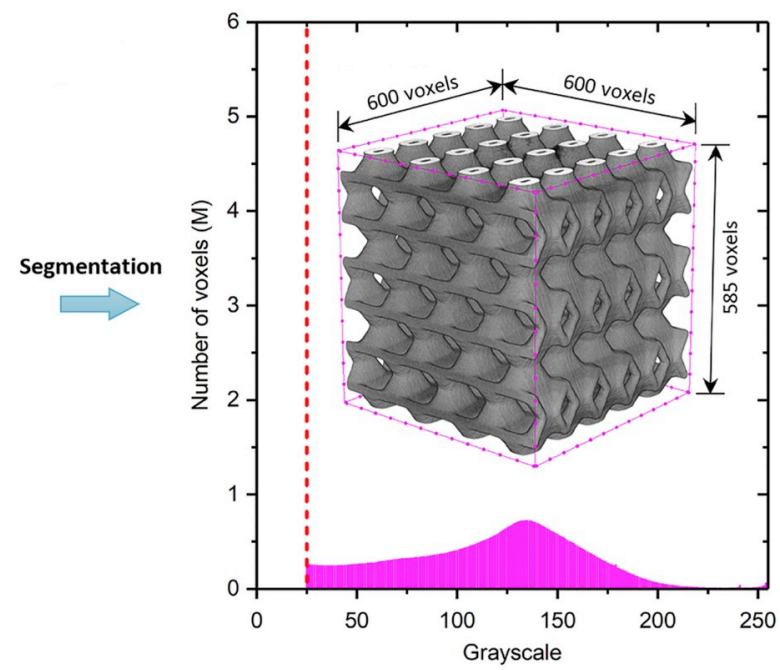

(b) Defined volume of interest and its histogram

Fig. 3. Cropped reference volume image, defined volume of interest and their histograms.

During the tests, large compressive deformations were imposed to the test specimen, therefore, a nearest subvolume offset strategy combined with a "preconditioned" match scheme was employed for incremental DVC analysis with enhanced accuracy and efficiency [24]. During volume image matching, a zero-mean normalized sum of the squared difference (ZNSSD) criterion was used for grayscale similarity measure. An advanced 3D inverse-compositional Gauss-Newton (ICGN) algorithm was adopted for subvoxel registration with better noiseproof performance. A layer-wise reliability guided displacement tracking (LW-RGDT) strategy was applied for displacement tracking and transfer [25]. The basic principle of incremental DVC analysis in this work including volume image matching, displacement transfer, and subvoxel registration is illustrated in Fig. 4. All DVC calculations were implemented using a self-coded academic software written in $\mathrm{C}++$ language on a laptop computer (Intel(R) Core(TM) i7-5500U CPU with $2.40 \mathrm{GHz}$ main frequency and $8 \mathrm{~GB}$ RAM).

\section{Results}

\subsection{Uncertainty assessment for DVC measurement}

Standard deviations (SDs) in displacement and strain fields 
(a) Incremental match strategy

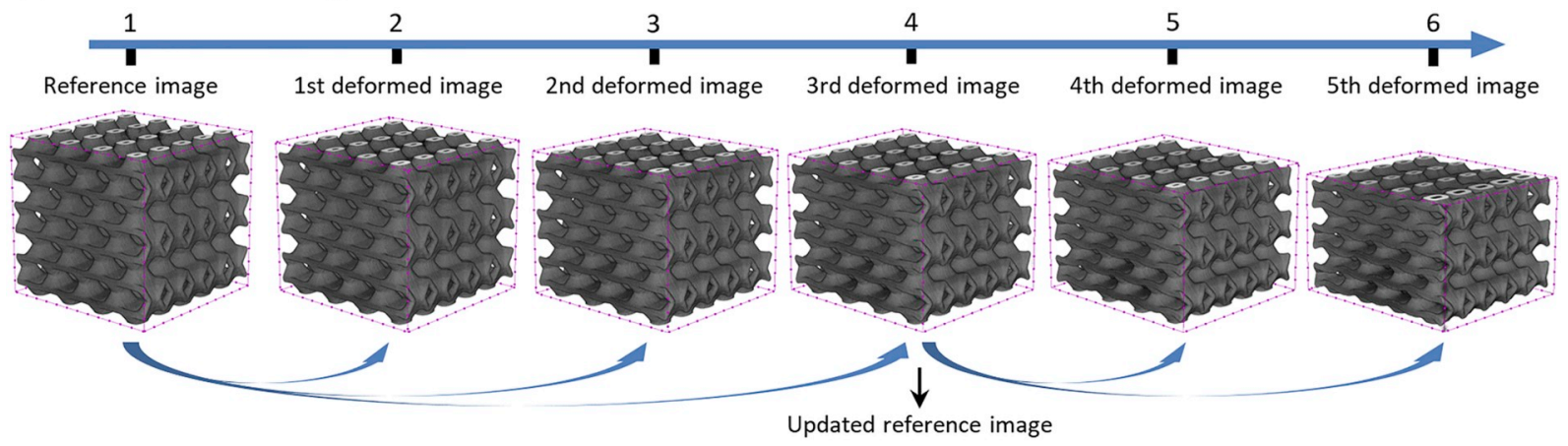

(b) Layer-wise RGDT strategy

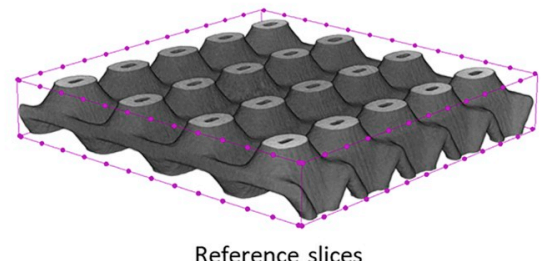

Reference slices

(c) 3D IC-GN algorithm

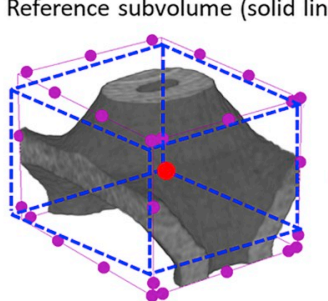

Warped reference subvolume (dash line)
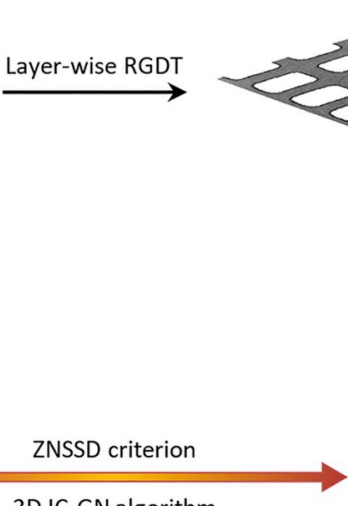

3D IC-GN algorithm
Calculation layer

Target subvolume (solid line)

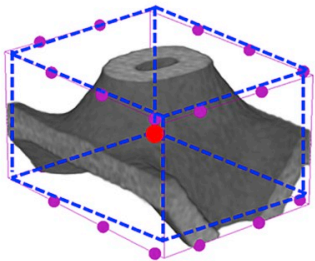

Updated target subvolume (dash line)

Fig. 4. Basic principle of incremental DVC analysis: (a) volume image matching; (b) displacement transfer; (c) subvoxel registration.

measured by DVC method using six different subvolume sizes in the rescan test of the 3D printed porous sample were exhibited in Fig. 5. Image noise and artifacts are two major factors causing DVC measurement uncertainty between two rescanned volume images [26,27]. As reported in the existing literature [28,29], the detrimental influence of image noise and artifacts can be evidently suppressed by using larger subvolume size, thus resulting in reduced SDs in displacements and strains. Specifically, as the subvolume size increases from $11 \times 11 \times 11$ to $61 \times 61 \times 61$ voxels, the SDs in displacements and strains decrease from 0.17 voxels to 0.02 voxels and from $4200 \mu \varepsilon$ to $500 \mu \varepsilon$, respectively. According to the baseline test, a subvolume size of $51 \times 51 \times 51$ voxels was chosen for subsequent DVC calculation in the step-wise uniaxial

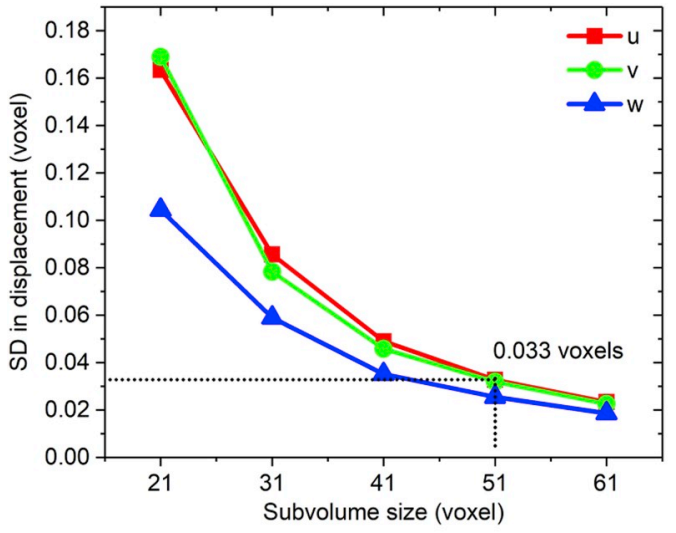

compression tests of the $3 \mathrm{D}$ printed porous sample to ensure a strain uncertainty less than $1000 \mu \varepsilon$. In this case, a displacement uncertainty of 0.033 voxels can be ensured as shown in the left graph.

\subsection{Internal deformation response}

Fig. 6 indicates the zero-mean normalized cross-correlation (ZNCC) coefficient fields measured by incremental DVC approach in the stepwise uniaxial compression test of the 3D printed porous sample. In general, higher ZNCC coefficient means a closer match between two volume images and hence more reliable displacement results. As shown in these graphs, the average ZNCC coefficient gradually reduces from

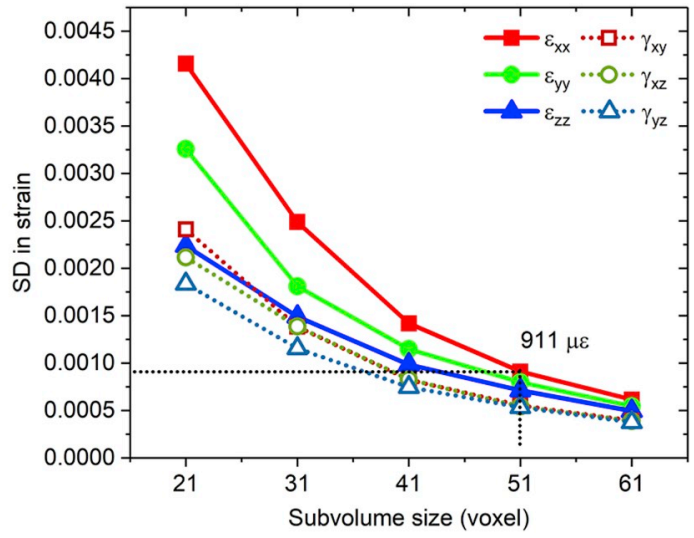

Fig. 5. Displacement and strain uncertainties of DVC measurement in the rescan test. 


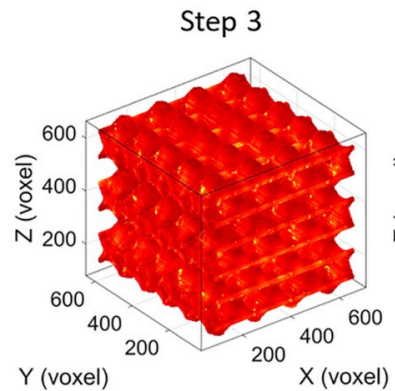

$Y($ voxel)

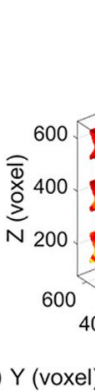

Average: 0.9877
Step 4

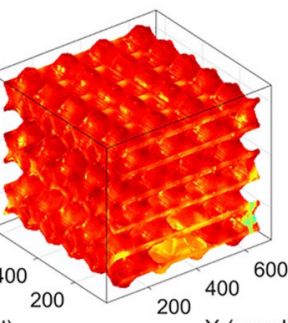

200

SD: 0.0041

Average: 0.9835

SD: 0.0063

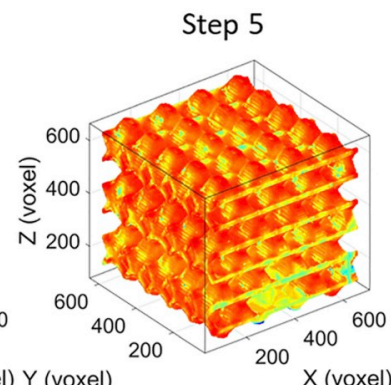

$X($ voxel) $Y($ voxel)

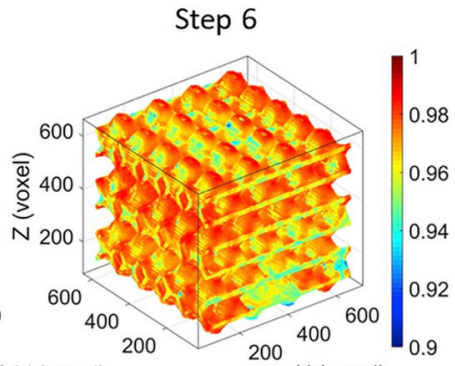

Average: 0.9698

SD: 0.0101

Fig. 6. DVC-measured ZNCC coefficient fields in the step-wise uniaxial compression test.
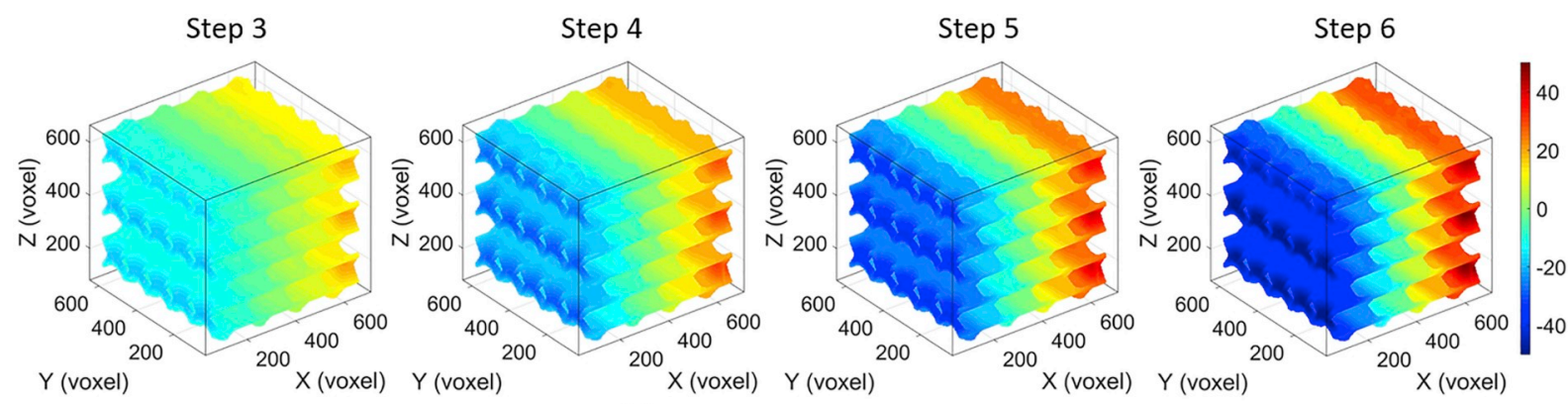

(a) $u$ displacement field
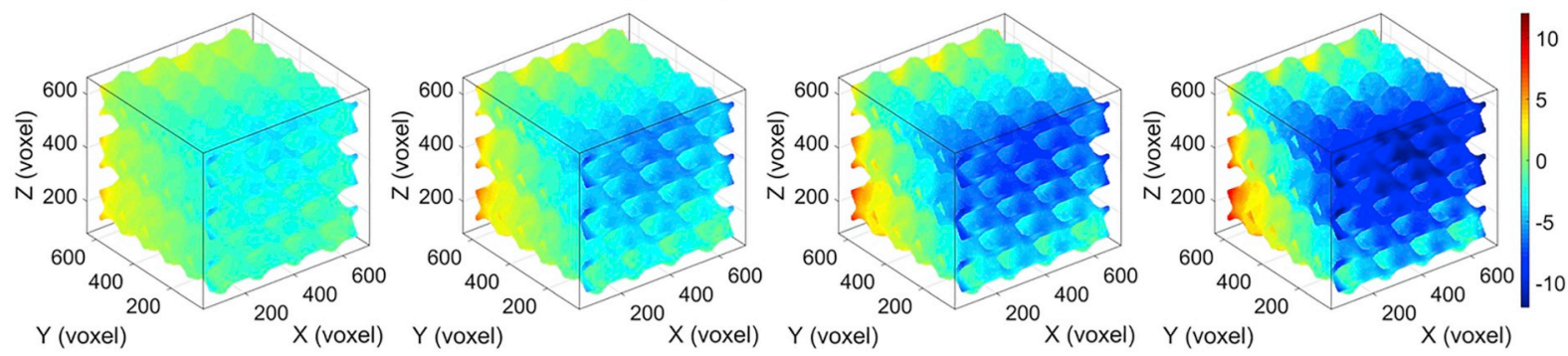

(b) $\vee$ displacement field
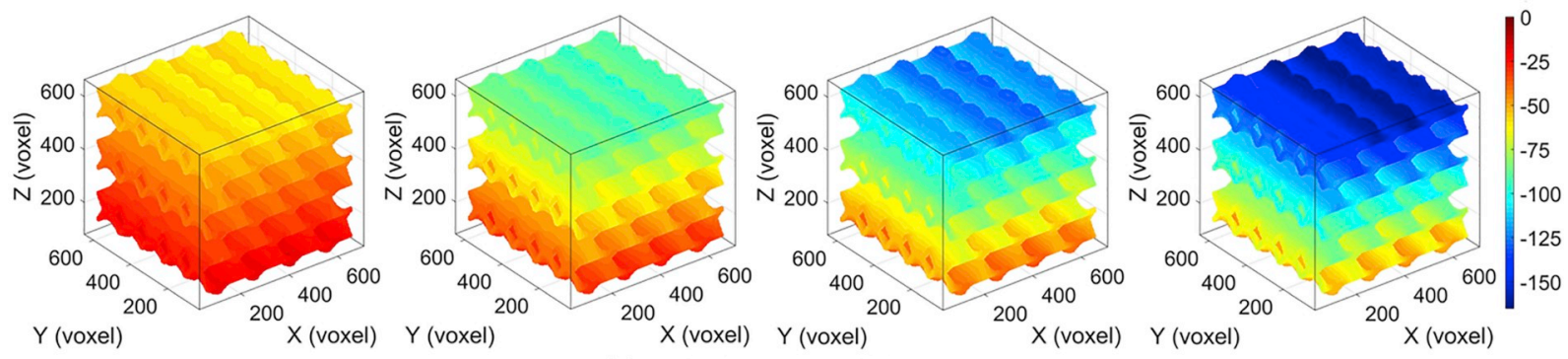

(c) $w$ displacement field

Fig. 7. DVC-measured $u, v$ and $w$ displacement fields in the step-wise uniaxial compression test.

0.9877 to 0.9698 with the increasing loading step. Note that the fullfield ZNCC coefficients evidently decrease in step 5 because updating reference volume image induces accumulated error in incremental DVC as shown in Fig. 4. In addition, due to the appearance of ring artifacts and beam hardening, ZNCC coefficients within several regions decrease at steps 5 and 6 . In each ZNCC coefficient field, the boundary zone of the 3D printed sample shows lower correlation coefficients than the center zone because of much fewer correlation points during displacement tracking. Despite these unfavorable factors, the lowest ZNCC coefficient in the last loading step is still higher than 0.9, indicating a reliable and robust deformation measurement during DVC analysis.

Fig. 7 illustrates the internal $u, v$ and $w$ displacement fields measured by the incremental DVC approach in the step-wise uniaxial compression test. The $w$ displacement fields indicate evident $z$-directional compression feature, and the maximal compressive motion within the VOI reaches $9 \mathrm{~mm}$ in the last loading step. The measured $u$ and $v$ displacement fields show different degrees of transversal expansions in each loading step. Specifically, $u$ field ranges from -45 to 45 voxels (nearly from $-2.49 \mathrm{~mm}$ to $2.49 \mathrm{~mm}$ ), while $v$ field exhibits a range of -12 to 12 voxels (nearly from $-0.66 \mathrm{~mm}$ to $0.66 \mathrm{~mm}$ ) in the last loading stage, thus reflecting diverse Poisson's ratios in $x$ and $y$ directions. This is mainly attributed to the different structural distributions of the 3D printed porous sample in xoz and yoz planes.

Based on the measured displacement fields, six Cauchy strain fields in each loading step can be estimated via the 3D PLS method. Fig. 8 depicts the internal $\varepsilon_{x x}, \varepsilon_{z z}$ and $\gamma_{x z}$ strain fields measured by the 

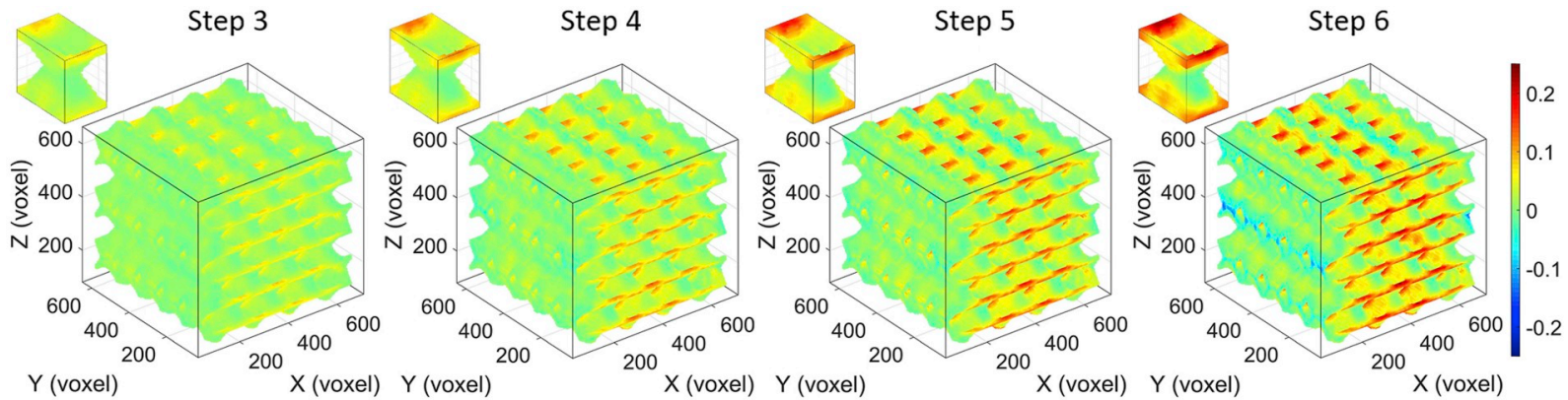

(a) $\varepsilon_{x x}$ strain field
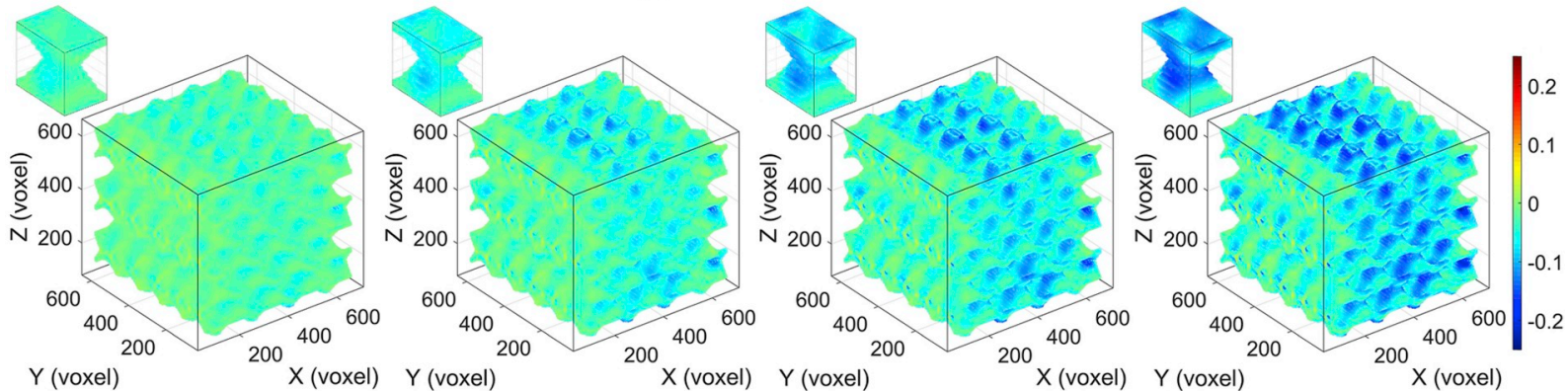

(b) $\varepsilon_{z z}$ strain field
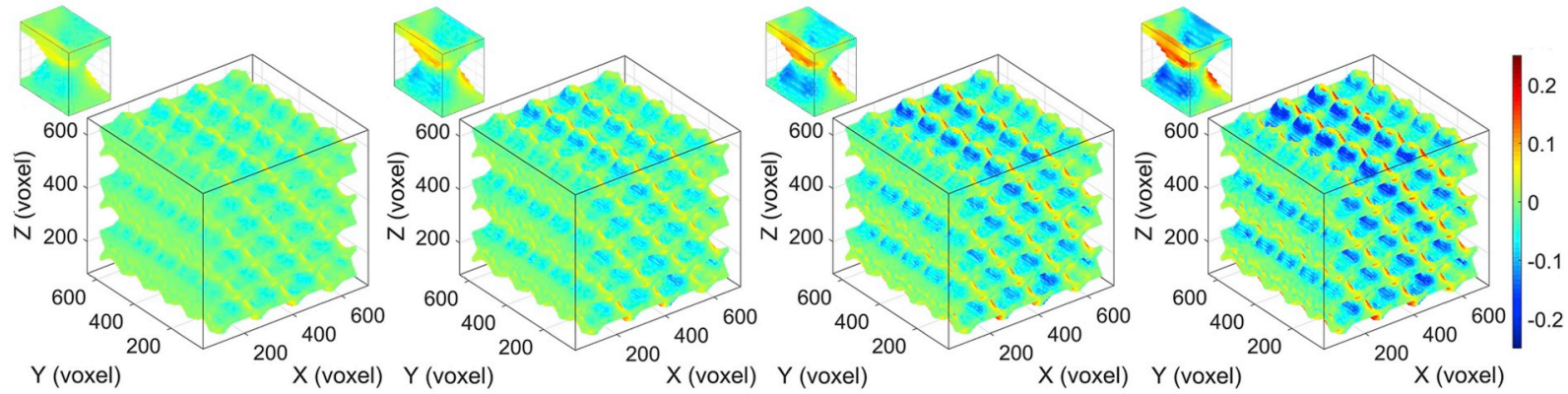

(c) $\gamma_{x z}$ strain field

Fig. 8. DVC-measured $\varepsilon_{x x}, \varepsilon_{z z}$ and $\gamma_{x z}$ strain fields in the step-wise uniaxial compression test.

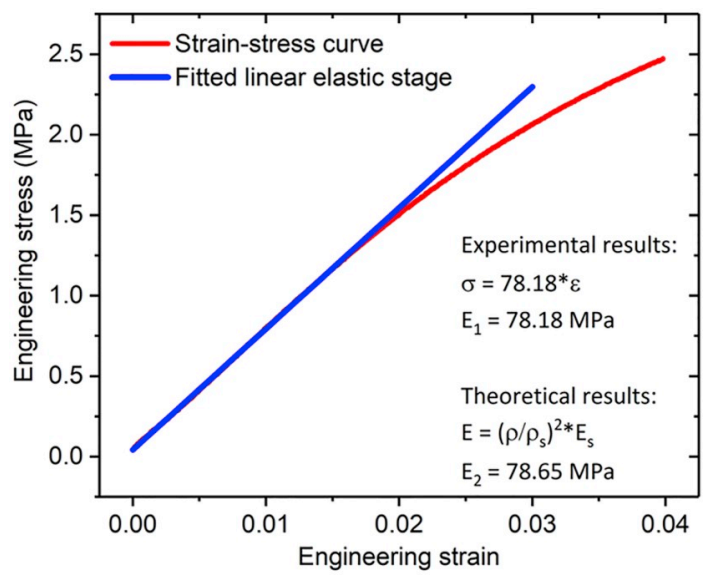

Fig. 9. Fitted linear elastic stage of the strain-stress curve in the step-wise uniaxial compression test.

incremental DVC approach in the step-wise uniaxial compression test. All the three strain fields exhibit local high strain domains in each loading step, and the strain localization becomes more significant as the compressive load increases. It is interesting to note that, from the first loading step on, local high strain zones in the three strain fields are periodically distributed within the porous sample. In each strain field, the high strain zones always appear at the same position within each unit cell as shown in the inserted small graphs. Specifically, high tensile strain zone locates on the top/bottom edge of each hourglass structure, while high compressive and shear strain zones are found on the side walls.

\subsection{Mechanical properties estimation}

Fig. 9 exhibits the fitted linear elastic stage of the engineering strain-stress curve in the step-wise uniaxial compression test. According to the porosity of the sample and the recorded strain-stress curve, the elastic modulus of the 3D printed porous sample was estimated from both experimental and theoretical perspectives. First, the engineering strain-stress curve of the first loading stage illustrated in Fig. 2 was extracted, and then the elastic modulus of the test sample was estimated as $78.18 \mathrm{MPa}$ from the fitted line. Second, the porosity of the undeformed test sample was estimated as $77.85 \%$, therefore, the elastic modulus of the porous sample was predicted as $78.65 \mathrm{MPa}$ according to the theoretical model of cellular solid $[30,31]$. By comparison, the relative error between the theoretical and experimental elastic moduli is less than $0.6 \%$, thus validating the reliability and practicability of the theoretical model.

Fig. 10 depicts the average normal strain, Poisson's ratio and volumetric strain of the 3D printed sample under step-wise compression. First, as the loading step increase, the three average normal strain components almost linearly increase with different slopes. In the last loading step, three average normal strains $\varepsilon_{x x}, \varepsilon_{y y}$ and $\varepsilon_{z z}$ reach 0.0316 , 

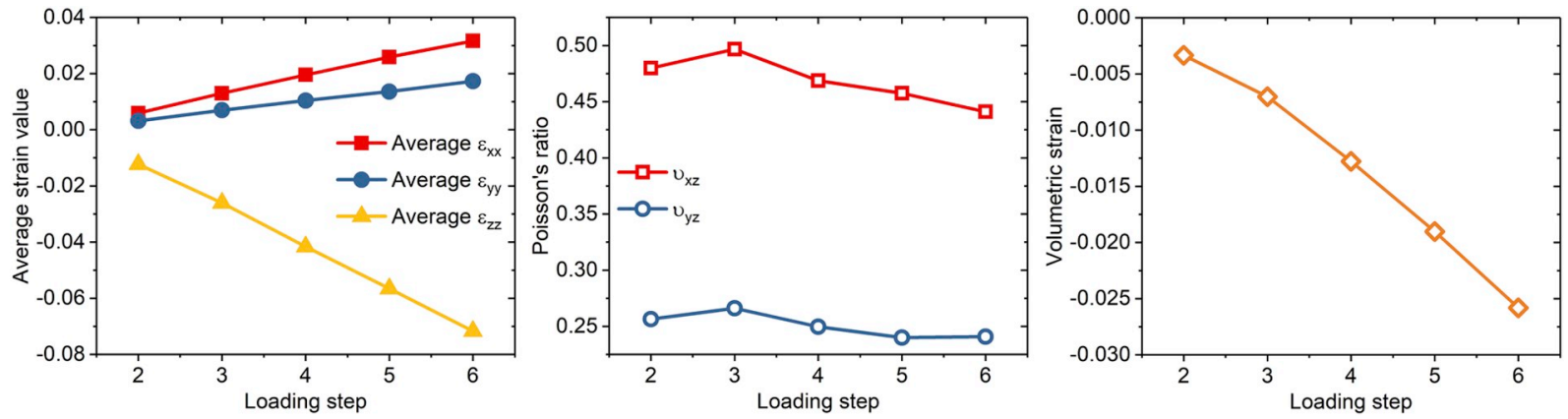

Fig. 10. Average normal strains, Poisson's ratios, and volumetric strain of the 3D printed sample under step-wise compression.
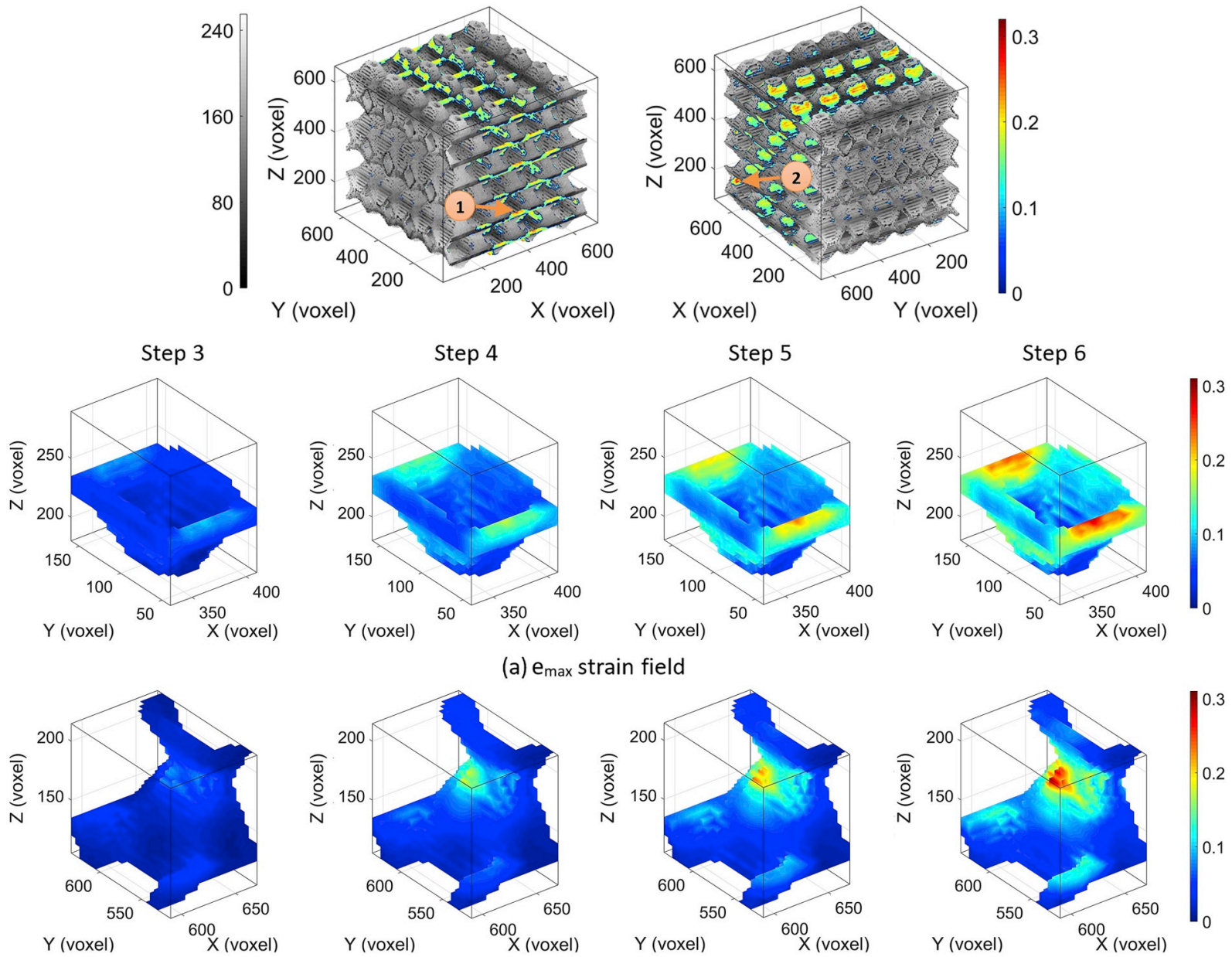

(b) $\gamma_{\max }$ strain field

Fig. 11. DVC-measured local evolutions of $\varepsilon_{\max }$ and $\gamma_{\max }$ strain fields in the step-wise uniaxial compression test.
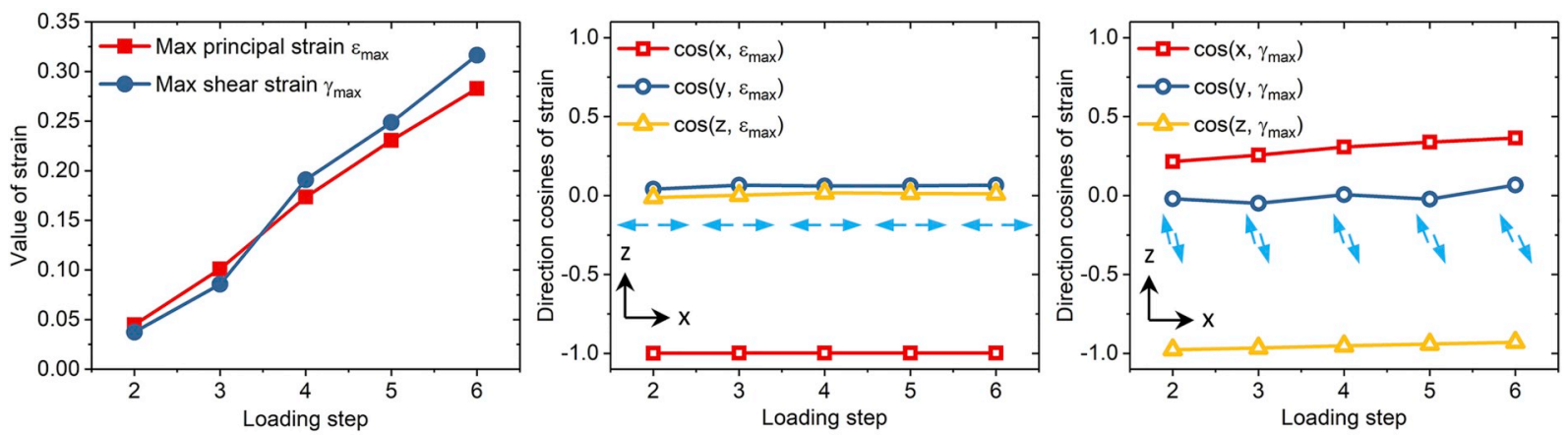

Fig. 12. Value and direction cosines of $\varepsilon_{\max }$ and $\gamma_{\max }$ strains in positions 1 and 2 in the step-wise uniaxial compression test. 

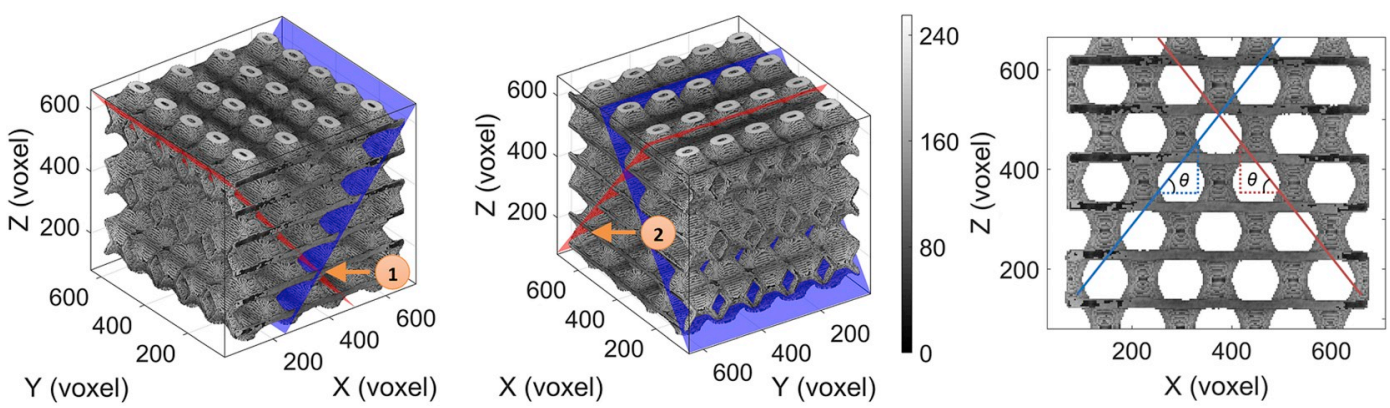

Fig. 13. Possible damage forms of the $3 D$ printed porous sample under step-wise uniaxial compression.

0.0173 and -0.0717 , respectively. Second, from the estimated normal strain results, Poisson's ratios $\nu_{x z}$ and $\nu_{y z}$ showing different values can be evaluated. Poisson's ratios are positive in all loading steps, with $\nu_{x z}$ ranging from 0.441 to 0.497 and $\nu_{y z}$ varying from 0.240 to 0.266 . Third, the variation trend of the volumetric strain approximates a straight line as the loading step increases. From loading step 2 on, the incremental volumetric strain gradually increases because the high strain zones enter the plastic state and hence show larger deformation response as shown in Fig. 8. It is interesting to note that, the difference in $\nu_{x z}$ and $\nu_{y z}$ is closely related to the geometric model and spatial distribution of unit cells within the test sample. This would play an important enlightening role in developing materials with adjustable Poisson's ratio based on this periodically stacked hourglass structure.

\section{Discussion}

Based on the measured six Cauchy strain fields, three principal strain and maximal shear strain fields in each loading step were extracted. Fig. 11 exhibits the full-field maximal principle strain $\varepsilon_{\max }$ and maximal shear strain $\gamma_{\max }$ in the last loading step mapped on the reference volume image. In these two graphs, small strain values less than 0.15 are hidden to highlight the distribution of high strain zones. It can be observed that local high strain zones are periodically distributed within the measured $\varepsilon_{\max }$ and $\gamma_{\max }$ fields. Positions 1 and 2 subjected to maximal $\varepsilon_{\max }$ and $\gamma_{\max }$ strains are marked in the two graphs, respectively. Specifically, position 1 located on the top edge of the unit cell is in the middle of the sample along the $x$-axis, while position 2 located on the side wall of the unit cell is in the corner of the sample. Fig. 11 also shows the local evolution of $\varepsilon_{\max }$ fields around position 1 and $\gamma_{\max }$ fields around position 2 in the step-wise compression test. The maximal $\varepsilon_{\max }$ and $\gamma_{\max }$ strains in positions 1 and 2 increase with the loading step and respectively approximate 0.3 and 0.32 in last loading step, which is close to the fracture elongation of the printing material listed in the inserted table in Fig. 1.

Fig. 12 illustrates the value and direction cosines of $\varepsilon_{\max }$ and $\gamma_{\max }$ strains in positions 1 and 2 in different loading steps. In the last loading step, the DVC-measured $\varepsilon_{\max }$ and $\gamma_{\max }$ strains at points 1 and 2 are 0.283 and 0.315 , respectively. As the loading step increases, the direction of $\varepsilon_{\max }$ strain at point 1 always keeps along the $x$-axis, while the angle between the direction of $\gamma_{\max }$ strain and the $x$-axis at point 2 decreases from $77^{\circ}$ to $68^{\circ}$. As the compressive load continues to increase, these two positions can be considered as the final failure points since their tensile/shear strains would first reach the fracture elongation of the printing material. According to the analysis of maximal principal and shear strains, specific damage forms and possible damage mechanism of the 3D printed porous sample can be reasonably deduced.

Fig. 13 depicts the possible damage forms of the 3D printed porous sample under uniaxial compression. The three planes passing through points 1 and 2 are marked as the potential rupture surface due to high $\varepsilon_{\max }$ and $\gamma_{\max }$ strains. Note that local high $\varepsilon_{\max }$ and $\gamma_{\max }$ strain zones appear alternately along the marked plane, therefore, the possible damage mechanism can be considered as the combined influence of localized high $\varepsilon_{\max }$ and $\gamma_{\max }$ strains. Also, one symmetric plane marked in the middle graph is considered as the possible failure route due to the symmetric structure of the 3D printed porous sample. In summary, due to the combined effects of localized high $\varepsilon_{\max }$ and $\gamma_{\max }$ strains on these staggered hourglass structures, the marked rupture surfaces would obliquely extend throughout the entire specimen. Because of the specific failure route, the tilt angle $\theta$ of these fracture planes approximates $53^{\circ}$ (arctan $\theta \approx 1.33$ ), which is directly related to the height-to-width aspect ratio $(5.5 / 4.15 \approx 1.33)$ of the unit cell.

\section{Conclusions}

In-situ X-ray CT imaging and DVC technique were used to map fullfield 3D internal deformation of a 3D printed porous structure subjected to step-wise uniaxial compressive loading. Based on DVC measurements, mechanical properties (including the elastic modulus and Poisson's ratios) of the test sample were estimated. Also, potential failure routes along with possible failure mechanism were discussed based on experimentally estimated local evolutions of maximal principle strain and maximal shear strain. Several valuable conclusions and perspectives are given as follows:

(1) In combination with in-situ X-ray CT imaging, DVC technique is able to detect global and local evolutions of internal full-field deformation within 3D printed polymer with complex structures in response to external loadings. Moreover, DVC measurement can locate positions subjected to large deformation and further facilitate the analysis of failure patterns and potential damage mechanism. In this manner, effective and reliable failure prediction can be realized via X-ray CT imaging and DVC measurement without destroying the test sample in practical experiments.

(2) Mechanical properties of the 3D printed porous structure estimated by DVC are related to its geometric structure visualized by X-ray CT. First, periodically distributed local high strain zones within the sample are attributed to the unique distribution of hourglass structures. Second, the difference in Poisson's ratios $\nu_{x z}$ and $\nu_{y z}$ is closely related to the geometric model and spatial distribution of unit cells within the sample. Third, the tilt angle of the predicted fracture planes approximately equals to the height-to-width aspect ratio of the unit cell. More interestingly, by correlating structural features with mechanical properties, possible design of 3D printed hourglass structures with tunable Poisson's ratio was inspired via simply changing the aspect ratio of the unit cell.

(3) Based on the established experimental framework, the structureproperty relationship of 3D printed structures can be well understood and theoretical models of cellular solid can be experimentally validated. Using these experimental results for specifying boundary condition and extracting constitutive parameters, FEM simulations aided by X-ray CT imaging could be performed with higher reliability. Furthermore, based on the initially designed structures, the shape optimization technique can be employed to further optimize 
structural layout via maximizing (or minimizing) given criteria (e.g., strength, toughness), thus facilitating design and fabrication of unique structures for new engineering applications.

\section{Data availability statement}

The raw/processed data required to reproduce these findings cannot be shared at this time due to technical or time limitations.

\section{Acknowledgements}

This work is supported by the National Key Research and Development Program of China (2018YFB0703500), and the National Natural Science Foundation of China $(11872009,11632010)$.

\section{References}

[1] B. Lu, D. Li, X. Tian, Development trends in additive manufacturing and 3D printing, Engineering 1 (1) (2015) 085-089, https://doi.org/10.15302/J-ENG2015012.

[2] A. Plessis, I. Yadroitsev, I. Yadroitsava, S.G. Le Roux, X-ray microcomputed tomography in additive manufacturing, Rev. Current Technol. Appl. 5 (3) (2018) 227-247, https://doi.org/10.1089/3dp.2018.0060.

[3] A. Thompson, I. Maskery, R.K. Leach, X-ray computed tomography for additive manufacturing: a review, Meas. Sci. Technol. 27 (7) (2016) 072001, https://doi. org/10.1088/0957-0233/27/7/072001.

[4] B. Wang, A.J. Benitez, F. Lossada, R. Merindol, A. Walther, Bioinspired mechanical gradients in cellulose nanofibril/polymer nanopapers, Angew. Chem. Int. Ed. 128 (20) (2016) 6070-6074, https://doi.org/10.1002/anie.201511512.

[5] Q. Zhang, J. Chen, Z. Zhao, H. Tan, X. Lin, W. Huang, Microstructure and anisotropic tensile behavior of laser additive manufactured TC21 titanium alloy, Mater. Sci. Eng. A 673 (2016) 204-212, https://doi.org/10.1016/j.msea.2016.07.040.

[6] N. Aliheidari, R. Tripuraneni, A. Ameli, S. Nadimpalli, Fracture resistance measurement of fused deposition modeling 3D printed polymers, Polym. Test. 60 (2017) 94-101, https://doi.org/10.1016/j.polymertesting.2017.03.016.

[7] Y. Song, Y. Li, W. Song, K. Yee, K.Y. Lee, V.L. Tagarielli, Measurements of the mechanical response of unidirectional 3D-printed PLA, Mater. Des. 123 (2017) 154-164, https://doi.org/10.1016/j.matdes.2017.03.051.

[8] D. Kokkinis, F. Bouville, A.R. Studart, 3D printing of materials with tunable failure via bioinspired mechanical gradients, Adv. Mater. 30 (19) (2018) 1705808, https:// doi.org/10.1002/adma.201705808.

[9] M. Schaffner, J.A. Faber, L. Pianegonda, P.A. Rühs, F. Coulter, A.R. Studart, 3D printing of robotic soft actuators with programmable bioinspired architectures, Nat. Commun. 9 (1) (2018) 878, https://doi.org/10.1038/s41467-018-03216-w.

[10] S. Bhandari, R. Lopez-Anido, Finite element analysis of thermoplastic polymer extrusion 3D printed material for mechanical property prediction, Addit. Manuf. 22 (2018) 187-196, https://doi.org/10.1016/j.addma.2018.05.009.

[11] T. Doktor, I. Kumpová, S. Wroński, M. Śniechowski, J. Tarasiuk, G. Forte, D. Kytýřr, Influence of printing and loading direction on mechanical response in 3D printed models of human trabecular bone, Acta Polytech. CTU Proc. 18 (2018) 24-27, https://doi.org/10.14311/APP.2018.18.0024.

[12] S. Kumar, B.L. Wardle, M.F. Arif, J. Ubaid, Stress reduction of 3D printed compliance-tailored multilayers, Adv. Eng. Mater. 20 (1) (2018) 1700883, https://doi. org/10.1002/adem.201700883.

[13] K. Genovese, Three-dimensional microscopic deformation measurements on cellular solids, J. Mech. Behav. Biomed. Mater. 60 (2016) 78-92, https://doi.org/10.1016/ j.jmbbm.2015.12.043.
[14] A. Sutradhar, J. Park, D. Carrau, M.J. Miller, Experimental validation of 3D printed patient-specific implants using digital image correlation and finite element analysis, Comput. Biol. Med. 52 (2014) 8-17, https://doi.org/10.1016/j.compbiomed.2014. 06.002.

[15] J.C.E. Mertens, K. Henderson, N.L. Cordes, R. Pacheco, X. Xiao, J.J. Williams, N. Chawla, B.M. Patterson, Analysis of thermal history effects on mechanical anisotropy of 3D-printed polymer matrix composites via in situ X-ray tomography, J. Mater. Sci. 52 (20) (2017) 12185-12206, https://doi.org/10.1007/s10853-0171339-4.

[16] S. Zhao, S.J. Li, S.G. Wang, W.T. Hou, Y. Li, L.C. Zhang, Y.L. Hao, R. Yang, R.D.K. Misra, L.E. Murr, Compressive and fatigue behavior of functionally graded Ti-6Al-4V meshes fabricated by electron beam melting, Acta Mater. 150 (2018) 1-15, https://doi.org/10.1016/j.actamat.2018.02.060.

[17] B. Pan, K. Qian, H. Xie, A. Asundi, Two-dimensional digital image correlation for inplane displacement and strain measurement: a review, Meas. Sci. Technol. 20 (6) (2009) 062001, https://doi.org/10.1088/0957-0233/20/6/062001.

[18] B. Pan, Digital image correlation for surface deformation measurement: historical developments, recent advances and future goals, Meas. Sci. Technol. 29 (8) (2018) 082001, https://doi.org/10.1088/1361-6501/aac55b.

[19] B.K. Bay, T.S. Smith, D.P. Fyhrie, M. Saad, Digital volume correlation: three-dimensional strain mapping using X-ray tomography, Exp. Mech. 39 (3) (1999) 217-226, https://doi.org/10.1007/BF02323555.

[20] B.K. Bay, Methods and applications of digital volume correlation, J. Strain Anal. Eng. Des. 43 (8) (2008) 745-760, https://doi.org/10.1243/03093247JSA436.

[21] A. Buljac, C. Jailin, A. Mendoza-Quispe, J. Neggers, T. Taillandier-Thomas, A. Bouterf, B. Smaniotto, F. Hild, S. Roux, Digital volume correlation: review of progress and challenges, Exp. Mech. 58 (5) (2018) 661-708, https://doi.org/10. 1007/s11340-018-0390-7.

[22] B. Pan, Bias error reduction of digital image correlation using Gaussian pre-filtering, Optic Laser. Eng. 51 (10) (2013) 1161-1167, https://doi.org/10.1016/j.optlaseng. 2013.04.009.

[23] L.K. Huang, M.J.J. Wang, Image thresholding by minimizing the measures of fuzziness, Pattern Recogn. 28 (1) (1995) 41-51, https://doi.org/10.1016/0031 3203(94)E0043-K.

[24] B. Wang, B. Pan, Incremental digital volume correlation method with nearest subvolume offset: an accurate and simple approach for large deformation measurement, Adv. Eng. Software 116 (2018) 80-88, https://doi.org/10.1016/j. advengsoft.2017.12.004

[25] B. Pan, B. Wang, A flexible and accurate digital volume correlation method for internal deformation measurement, Meas. Sci. Technol. 28 (10) (2017) 105007 https://doi.org/10.1088/1361-6501/aa7e8a.

[26] M.J. Murphy, Z. Wei, M. Fatyga, J. Williamson, M. Anscher, T. Wallace, E. Weiss, How does CT image noise affect 3D deformable image registration for image-guided radiotherapy planning? Med. Phys. 35 (3) (2008) 1145-1153, https://doi.org/10. $1118 / 1.2837292$

[27] N. Limodin, J. Réthoré, J. Adrien, J.Y. Buffière, F. Hild, S. Roux, Analysis and artifact correction for volume correlation measurements using tomographic images from a laboratory X-ray source, Exp. Mech. 51 (6) (2011) 959-970, https://doi.org/ 10.1007/s11340-010-9397-4.

[28] B. Pan, H. Xie, Z. Wang, K. Qian, Z. Wang, Study on subset size selection in digital image correlation for speckle patterns, Optic Express 16 (10) (2008) 7037-7048, https://doi.org/10.1364/OE.16.007037.

[29] M. Bornert, F. Brémand, P. Doumalin, J.C. Dupré, M. Fazzini, M. Grédiac, F. Hild, S. Mistou, J. Molimard, J.J. Orteu, L. Robert, Y. Surrel, P. Vacher, B. Wattrisse, Assessment of digital image correlation measurement errors: methodology and results, Exp. Mech. 49 (3) (2009) 353-370, https://doi.org/10.1007/s11340-0089204-7.

[30] M.F. Ashby, R.F.M. Medalist, The mechanical properties of cellular solids, Metall. Trans. A. 14 (9) (1983) 1755-1769, https://doi.org/10.1007/BF02645546.

[31] L.J. Gibson, M.F. Ashby, Cellular Solids: Structure and Properties, Cambridge university press, 1999 13: 9780521499118. 